\title{
Summary of the Effort to Use Active- induced Time Correlation Techniques to Measure the Enrichment of HEU
}

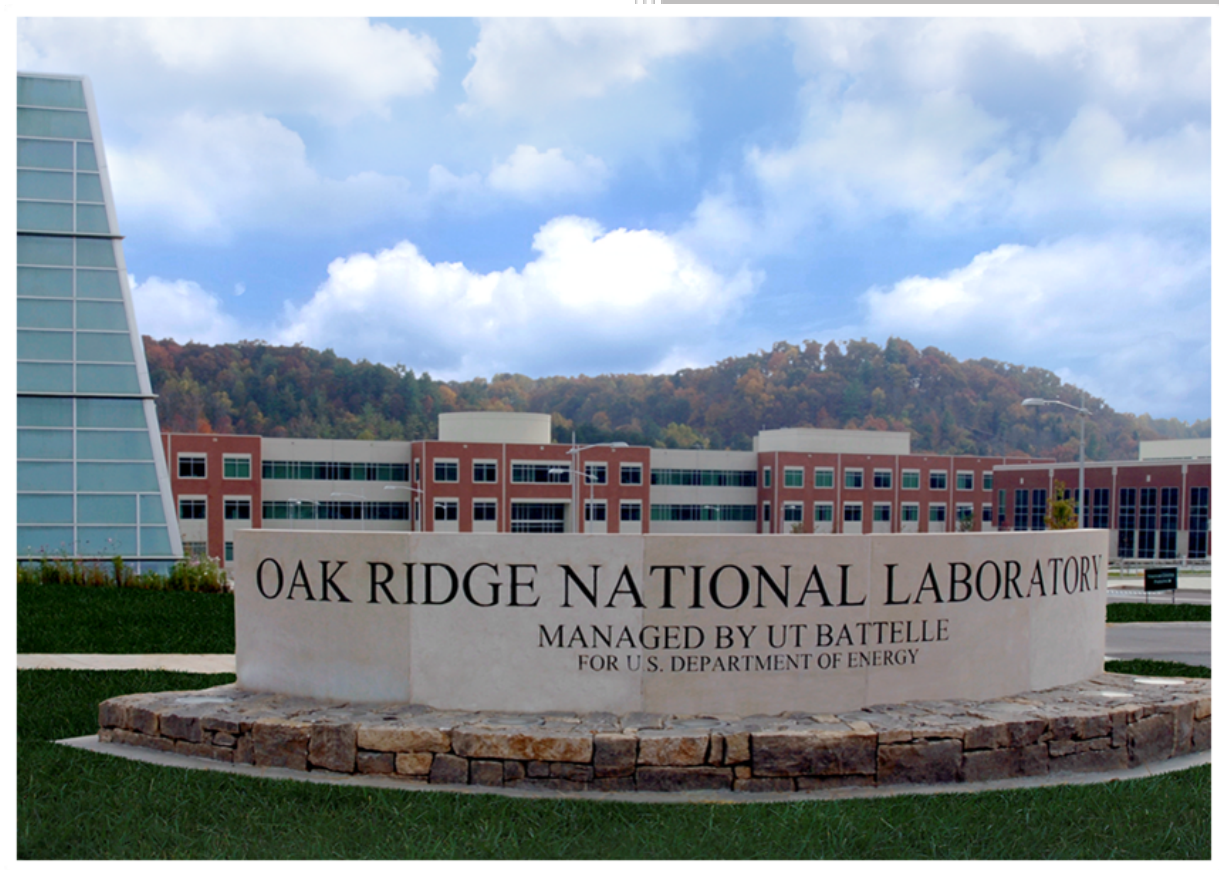

Approved for public release.

Distribution is unlimited.

Seth McConchie Jason Crye Kirsten Peña Eric Sword John Mihalczo

September 2015 


\title{
DOCUMENT AVAILABILITY
}

Reports produced after January 1, 1996, are generally available free via US Department of Energy (DOE) SciTech Connect.

Website http://www.osti.gov/scitech/

Reports produced before January 1, 1996, may be purchased by members of the public from the following source:

National Technical Information Service

5285 Port Royal Road

Springfield, VA 22161

Telephone 703-605-6000 (1-800-553-6847)

TDD 703-487-4639

Fax 703-605-6900

E-mail info@ntis.gov

Website http://www.ntis.gov/help/ordermethods.aspx

Reports are available to DOE employees, DOE contractors, Energy Technology Data Exchange representatives, and International Nuclear Information System representatives from the following source:

Office of Scientific and Technical Information

PO Box 62

Oak Ridge, TN 37831

Telephone 865-576-8401

Fax 865-576-5728

E-mail reports@osti.gov

Website http://www.osti.gov/contact.html

\begin{abstract}
This report was prepared as an account of work sponsored by an agency of the United States Government. Neither the United States Government nor any agency thereof, nor any of their employees, makes any warranty, express or implied, or assumes any legal liability or responsibility for the accuracy, completeness, or usefulness of any information, apparatus, product, or process disclosed, or represents that its use would not infringe privately owned rights. Reference herein to any specific commercial product, process, or service by trade name, trademark, manufacturer, or otherwise, does not necessarily constitute or imply its endorsement, recommendation, or favoring by the United States Government or any agency thereof. The views and opinions of authors expressed herein do not necessarily state or reflect those of the United States Government or any agency thereof.
\end{abstract}




\title{
Summary of the Effort to Use Active-induced Time Correlation Techniques to Measure the Enrichment of HEU
}

\author{
Seth McConchie ${ }^{1}$ \\ Jason Crye ${ }^{2}$ \\ Kirsten Peña ${ }^{2}$ \\ Eric Sword \\ John Mihalczo
}

30 September 2015

OAK RIDGE NATIONAL LABORATORY

Oak Ridge, Tennessee 37831-6283 managed by

UT-BATTELLE, LLC

for the

U.S. DEPARTMENT OF ENERGY

under contract DE-AC05-00OR22725

\footnotetext{
${ }^{1}$ PI Contact information: mcconchiesm@ornl.gov, 1-865-576-9376

${ }^{2}$ University of Tennessee Ph.D. students on this project
} 
This page intentionally left blank. 


\section{EXECUTIVE SUMMARY}

This document summarizes the effort to use active-induced time correlation techniques to measure the enrichment of bulk quantities of enriched uranium. In summary, these techniques use an external source to initiate fission chains, and the time distribution of the detected fission chain neutrons is sensitive to the fissile material enrichment. The number of neutrons emitted from a chain is driven by the multiplication of the item, and the enrichment is closely coupled to the multiplication of the item. As the enrichment increases (decreases), the multiplication increases (decreases) if the geometry is held constant. The time distribution of fission chain neutrons is a complex function of the enrichment and material configuration. The enrichment contributes to the probability of a subsequent fission in a chain via the likelihood of fissioning on an even-numbered isotope versus an odd-numbered isotope. The material configuration contributes to the same probability via solid angle effects for neutrons inducing subsequent fissions and the presence of any moderating material. To simplify the ability to accurately measure the enrichment, an associated particle imaging (API) D-T neutron generator and an array of plastic scintillators are used to simultaneously image the item and detect the fission chain neutrons. The image is used to significantly limit the space of enrichment and material configuration and enable the enrichment to be determined unambiguously.

The principal technical objective for this project has been to investigate the ability to determine the enrichment from shielded bulk enriched uranium using an API D-T neutron generator and an array of plastic scintillators. While the technique permits a deep probe of the item and the emitted neutrons more likely to be detected through high- $Z$ shielding, the analysis to determine the enrichment is significantly more complex than comparing the one or more gamma signatures. The complexity is due to full three-dimensional nature of the analysis to improve the accuracy of the enrichment determination. The most important accomplishments of this project are the:

1. Demonstration of the ability to accurately measure the enrichment of a $93.2 \mathrm{wt} \%$ highly enriched uranium (HEU) annular storage casting within $2.5 \mathrm{~cm}$ of depleted uranium (DU) shielding without calibration standard.

2. Demonstration of the ability to distinguish a $93.2 \mathrm{wt} \%$ HEU casting and DU storage casting shielded by $2.5 \mathrm{~cm}$ of DU shielding within two minutes at a $3 \sigma$ confidence level.

3. Development of a generalized iterative algorithm for enrichment determination requiring no geometrical simplifications (though simplifications may be made to reduce computational time).

The scope of future work is:

1. Perform measurements of castings with enrichments between DU and 93.2 wt\%. These measurements would enable a complete investigation of the time correlation observable's dependence on enrichment and potentially providing 
benchmarking data for future measurements. Other users of the Y-12 National

Security Complex's Nuclear Detector and Sensor Test Center would also benefit from the availability of multiple enrichment uranium castings.

2. Improve the ability to predict the active-induced time correlations to strengthen the tools within the enrichment determination algorithm.

Accurately predicting the time distribution of events has been a challenge. Previously Oak Ridge National Laboratory has successfully modeled ${ }^{252} \mathrm{Cf}$ sources and small $(2.5 \times 2.5 \times 15-\mathrm{cm}$ detectors $)$ closely coupled to the item. As the detector scale has been increased $(27 \times 27 \times 5.0 \mathrm{~cm})$ and detectors have been placed farther from the item, time-of-flight effects are revealing discrepancies with the modeling that had not been observed previously. This effort includes using the newly developed fission model codes in which the physics of the fission neutron and gamma emission has been improved. Additionally, detector response would also be addressed to correctly model the light production in a large detector.

3. Develop the algorithms to translate the fast neutron image to a format for use in the radiation transport code. Currently there is limited automated capability to translate a fast neutron image into a format suitable for performing three-dimensional radiation transport. This step is necessary for the iterative enrichment determination algorithm to function without human intervention. This effort would use the OpenCV toolkit or a similar toolkit.

4. Investigate further improvements to the enrichment determination algorithm. The enrichment determination algorithm that was developed in this project is generalized to allow for any configuration of materials. As a result, it is an iterative algorithm that varies the enrichment using the measured geometry until the calculated observables match the measured observables. One potential path for reducing the complexity is to investigate the dependence of the coupling probability for inducing the first fission on enrichment. Existing image reconstruction algorithms could then be applied to extract the coupling probability as a function of position in the item and therefore the enrichment.

5. Assess the application of the enrichment determination algorithm on classified uranium shapes. This technique has potential application to areas such as arms control and treaty verification. 


\section{TABLE OF Contents}

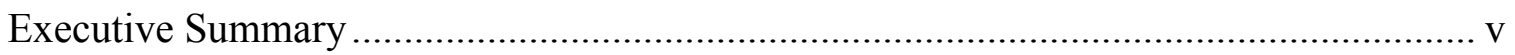

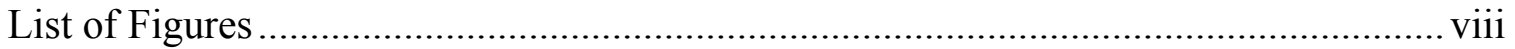

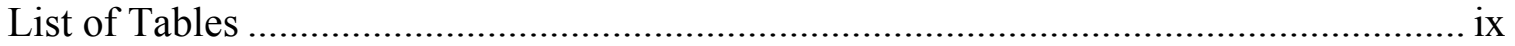

Acronyms and Abbreviations .................................................................................... $\mathrm{x}$

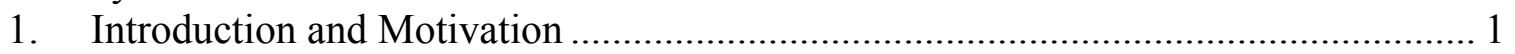

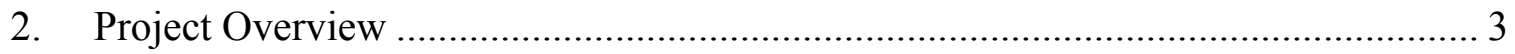

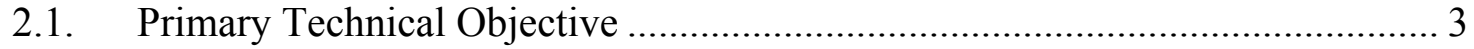

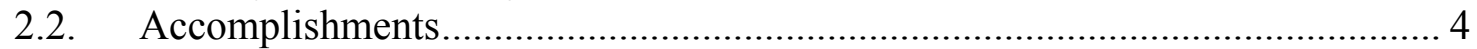

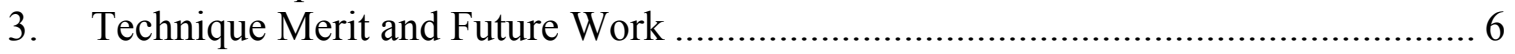




\section{LIST OF FIGURES}

Figure 1. Nuclear Materials Identification System (NMIS) setup at the Nuclear Detector and Sensor Test Center with a DU casting and multiple DU shields.

Figure 2. Demonstration of the sensitivity of the active-induced neutron coincidences to enrichment using a $93.2 \mathrm{wt} \%$ HEU casting, DU casting, and $2.5 \mathrm{~cm}$ DU shielding.

Figure 3. Enrichment determination algorithm flowchart based upon the multiplication.................................................................................. 8

Figure 4. Demonstration of the discrepancy when comparing modeled and measured time correlation distributions 


\section{LiST OF TABLES}

Table 1. List of deliverables with descriptions and cross-references to the accomplishment list.

Table 2. Comparison of measured multiplications for a $93.2 \mathrm{wt} \% \mathrm{HEU}$ casting, DU casting, and various amounts of DU shielding. Uncertainties represent the $3 \sigma$ error bar. 


\section{ACRONYMS AND ABbREVIATIONS}

$\begin{array}{ll}\text { API } & \begin{array}{l}\text { associated particle imaging } \\ \text { depleted uranium } \\ \text { enriched uranium }\end{array} \\ \text { EU } & \begin{array}{l}\text { fiscal year } \\ \text { graphics processing unit } \\ \text { FY }\end{array} \\ \text { GPU } & \text { Mighly enriched uranium } \\ \text { HEU } & \text { Nuclear Detector and Sensor Test Center } \\ \text { MCNP } & \text { Nuclear Materials Identification System } \\ \text { NDSTC } & \text { Oak Ridge National Laboratory } \\ \text { NMIS } & \text { Y-12 National Security Complex }\end{array}$




\section{INTRODUCTION AND MOTIVATION}

Quantifying the enrichment of bulk uranium using non-destructive assay is an important but challenging problem. One of the traditionally considered attributes for monitored dismantlement is fissile material enrichment for which passive gamma spectrometry is the standard assay method. Passive gamma spectrometry has been demonstrated for quantifying plutonium enrichment in various exercises, including the Warhead Monitored Dismantlement exercise organized by the Office of Nuclear Verification in 2011. This technique uses the ${ }^{239} \mathrm{Pu}$ and ${ }^{240} \mathrm{Pu}$ lines in the $635-642 \mathrm{keV}$ energy region to estimate isotopic ratio of ${ }^{239} \mathrm{Pu}$ to ${ }^{240} \mathrm{Pu}$. The primary advantages these gamma emissions are (1) they are sufficiently close in energy that self-attenuation and detector efficiency corrections are negligible, and (2) they penetrate more high-Z material than other higher intensity, lower energy gammas. These advantages do not exist for uranium. The most intense ${ }^{235} \mathrm{U}$ and ${ }^{238} \mathrm{U}$ gamma emissions have energies of $186 \mathrm{keV}$ and $1001 \mathrm{keV}$, respectively, which differ enough to require self-attenuation and detector efficiency corrections. There are gamma emissions sufficiently close in energy to avoid these corrections, but they are below $100 \mathrm{keV}$. These gammas are heavily self-attenuated and are easily shielded with a thin layer of high-Z shielding.

These deficiencies are mitigated partly by neutron or gamma emissions with higher energy. An alternative technique is to measure the delayed neutron precursors using a pulsed source and moderated thermal neutron detectors. Delayed neutrons have an energy scale of several hundred $\mathrm{keV}$ such that they can be considered a deeper probe of bulk fissile material than gamma emissions. Additionally, the delayed neutron population as a function of time is a function of the enrichment. This technique is inherently challenging to apply on complex geometries with shielded uranium. Prompt fission neutrons and gamma emissions can also be considered a deeper probe of the fissile material but present their own interpretational challenges. Fission neutrons and gamma rays are correlated in time, such that neutrons and gamma rays from the same fission chain appear in the detector event stream in bursts. The number of events in these bursts manifests from the self-multiplication (hereafter multiplication) of the geometry and is a non-trivial function of the enrichment. The challenge is complicated additionally by the fact that the multiplication is degenerate in both the enrichment and configuration of materials. Two different material configurations and different enrichments can result in the same observed neutron and gamma singles and coincidences (i.e., doubles, triples, etc.). However, if the relative amount of ${ }^{235} \mathrm{U}$ to ${ }^{238} \mathrm{U}$ is increased in configuration while the rest of the configuration remains fixed, the multiplication will increase. The change will be evident in the fission chain observables.

The measurement challenge could be potentially reduced if the geometry could be measured and used to interpret the enrichment dependent observables regardless of the technique. Imaging techniques such as x-ray imaging or active fast neutron imaging are well suited for measuring the geometry. While x-ray imaging performs on the submillimeter scale, intense, high-energy sources are required to penetrate high- $Z$ shielding and the bulk fissile material itself. Fast neutron imaging can probe high-Z materials more efficiently (i.e., more transmitted particles per incident particle) than X-ray imaging, but 
the technique probes low- $Z$ materials less efficiently. Thus, active fast neutron imaging may be considered a complementary technique to $\mathrm{x}$-ray imaging. State-of-the-art transportable fast neutron imaging systems have spatial resolutions on the order of $5 \mathrm{~mm}$, corresponding well to the size scale of a multiplying $(M \geq 1.5)$ bulk uranium configuration. Oak Ridge National Laboratory (ORNL) recognized that active fast neutron imaging based on time correlations could determine the geometry and measure the induced fission chain neutrons and gammas in the same measurement. ORNL had previously developed expertise in transportable active fast neutron imaging based on time correlations. The commonly known ORNL-developed instrument that employs this technique is the Nuclear Materials Identification System (NMIS).

NMIS uses an instrumented associated particle imaging D-T neutron generator and an array of plastic scintillator detectors. The alpha particle emitted from the D-T reaction in the neutron generator is time and directionally tagged, indicating the emission time and direction of travel for the corresponding $14 \mathrm{MeV}$ neutron. For sufficient pixelization of the alpha detector and detector array, a total attenuation map, or image, can be made for an item using the directly transmitted neutrons. If the item is circularly symmetric or is rotated, then a tomographic reconstruction gives the attenuation throughout the item. A study of the reconstruction enables a coarse identification of materials, such as uranium, iron, lead, and polyethylene or other low-Z materials. For those neutrons that induce fission in the item, the resulting fission neutrons and gamma rays are correlated to an alpha and therefore can be imaged similarly to the directly transmitted neutrons. For a multiplying item, the fission chain neutrons and gamma rays will also be correlated to an alpha, enabling the ability to distinguish between fissile and fissionable material.

As the neutrons and gamma emissions are attenuated differently through the item, the analysis is simplified if the neutron and gamma contributions can be distinguished. The ability to distinguish neutrons and gamma rays can be accomplished via time-of-flight on the nanosecond scale or by using detectors with pulse shape discrimination capability. The instrumentation is arguably simpler when employing only time-of-flight, but the detector array must be set tens of centimeters away from the item to enable the fission neutrons spanning several $\mathrm{MeV}$ in energies to arrive after the majority of the gamma rays. As an example with the NMIS instrumentation, a detector array with an intrinsic efficiency of $30 \%$ must cover $17 \%$ of the solid angle to accomplish an absolute efficiency of $5 \%$. For a distance of $50 \mathrm{~cm}$ between the center of the item and the front of the detector array, the surface area must be $5300 \mathrm{~cm}^{2}$, which drives the scale of the detector footprint.

In this project, the fission chain neutron singles and coincidences were used as the observables because they provide a deeper probe of the fissile material. The most significant challenges with interpreting the induced fission chain neutrons are (1) establishing the sensitivity of the fission chain neutron singles and coincidences to the enrichment, and (2) extracting a meaningful enrichment from the observables. The subsequent challenge is to quantify the enrichment within a short analysis time of up to a couple of minutes. The primary objective of this document is to summarize the effort to address these challenges with a modeling suite with radiation transport simulations, 
active time correlation measurements on configurations of enriched uranium (EU), and algorithm development for enrichment determination.

\section{Project OvervieW}

\subsection{Primary Technical Objective}

The primary technical objective of this project was to evaluate active-induced time correlation signatures to determine the enrichment of shielded bulk uranium. This objective was accomplished with a series of steps that can be outlined as:

1. Develop the measurement plan

a. What enriched uranium configurations can be measured to evaluate the technique?

b. Where would the measurements be performed?

2. Perform a modeling suite of radiation transport simulations

a. What is the representative suite of simulations?

b. How sensitive is the technique to the enrichment?

c. How much measurement time is needed to perform a measurement?

3. Develop the enrichment determination algorithm(s)

a. What are the potential algorithms for deriving enrichment from the time correlation observables?

b. How complex are the algorithms and how quickly would they execute?

4. Perform the measurements and data analysis

a. How well do the data match the simulations?

b. What else may have been learned from the measurements?

The scope of the simulations was driven by the measurement plan. During the proposal process, ORNL proposed to perform measurements at the Nuclear Detector and Sensor Test Center (NDSTC) at the Y-12 National Security Complex (Y-12). Y-12 had provided a $93.2 \mathrm{wt} \%$ highly enriched urnanium (HEU) 161 annular storage casting and a depleted uranium (DU) casting for prior ORNL measurements and indicated it could potentially provide castings of other enrichments. As a result, the majority of the simulations were centered on an EU casting with shielding of various thicknesses and with the material compositions of polyethylene, steel, lead, and DU.

The primary focus of the measurements was placed on the DU shielding for a couple of reasons. First, the $14 \mathrm{MeV}$ neutron attenuations of polyethylene, lead, and steel are distinguishable from uranium. If the observed neutron singles and coincidences indicate the presence of a multiplying material, then the EU can be localized using the transmission image. ${ }^{235} \mathrm{U}$ and ${ }^{238} \mathrm{U}$ have nearly the same $14 \mathrm{MeV}$ neutron attenuation, such that DU and EU cannot be distinguished by transmission alone. Thus, EU annuli shielded by DU annuli represented an intermediate level of difficulty for investigating the limits of the active time correlation method. ORNL purchased the DU shields for use in 
the NDSTC measurements with any EU castings that Y-12 could provide. Second, the DU shields are excellent high-Z shielding to prevent the detection of ${ }^{235} \mathrm{U}$ decay gamma signatures. Any enrichment characterization capability established with time correlation methods fills a capability gap associated with passive gamma spectrometry and high-Z shielding.

\subsection{Accomplishments}

The project accomplishments are partitioned according to each fiscal year and may be listed as:

1. Establishment of the enrichment on multiplication in fissile configurations (fiscal year (FY)09/FY10)

2. Completion of the radiation transport simulations with EU metal configurations shielded by DU (FY09/FY10)

a. Completion of the criticality simulations to investigate multiplication

b. Completion of the time-correlation simulations (neutron singles and neutron coincidences correlated to $14 \mathrm{MeV}$ neutrons) with varying enrichment

3. Conceptualization of the algorithm for enrichment determination and initial work (FY09/FY10)

a. Concept development for faster Monte Carlo transport

b. Implementation of fission chain probability calculation on the graphics processing unit platform

4. Development of enrichment determination algorithm (FY11/FY12)

a. Extension of point kinetics model for coupled sources to estimate multiplication from time correlation observables

b. Initial development of Monte Carlo 2D transport algorithm

c. Implementation of algorithm to translate fast neutron image to surfaces for use in Monte Carlo transport

d. Investigation of existing Monte Carlo tools performance

5. Investigation of limitations of active-induced time correlations (FY11)

a. Analysis of configurations varying in scale

b. Assessment of minimum possible enrichment from a measurement

6. Completion of the measurements of $93.2 \mathrm{wt} \%$ HEU shielded by DU and analysis of the data (FY12)

The list of deliverables that documents the details of these accomplishments is given in Table 1. 
Table 1. List of deliverables with descriptions and cross-references to the accomplishment list.

\begin{tabular}{|c|c|c|c|}
\hline Deliverable & FY & Description & Accomplishments \\
\hline $\begin{array}{l}\text { Methodology for } \\
\text { Enrichment } \\
\text { Determination of DU } \\
\text { Shielded HEU } \\
\text { Assemblies Using Time } \\
\text { Correlation } \\
\text { Techniques with } \\
\text { Source Induced Fission } \\
\text { Chains }\end{array}$ & FY09/FY10 & $\begin{array}{l}\text { Describes the concept for } \\
\text { enrichment determination } \\
\text { using active-induced time } \\
\text { correlation signatures and the } \\
\text { modeling to investigate the } \\
\text { sensitivity of measurement } \\
\text { observables to enrichment }\end{array}$ & $1,2,3$ \\
\hline $\begin{array}{l}\text { Analysis Studies for } \\
\text { Algorithm } \\
\text { Enhancements to } \\
\text { Determine the } \\
\text { Enrichment of HEU } \\
\text { Assemblies Shielded by } \\
\text { DU Using Time } \\
\text { Correlation } \\
\text { Techniques }\end{array}$ & FY11 & $\begin{array}{l}\text { Describes the development of } \\
\text { the enrichment determination } \\
\text { algorithm in preparation for the } \\
\text { measurements }\end{array}$ & 4,5 \\
\hline $\begin{array}{l}\text { Algorithm } \\
\text { Refinements Based } \\
\text { Upon the Results of the } \\
\text { Enrichment } \\
\text { Measurements }\end{array}$ & FY12 & $\begin{array}{l}\text { Describes refinements to the } \\
\text { algorithm based upon } \\
\text { measurement results }\end{array}$ & 4 \\
\hline $\begin{array}{l}\text { Measurements for the } \\
\text { Enrichment } \\
\text { Determination of HEU } \\
\text { Assemblies Shielded by } \\
\text { DU Using Time } \\
\text { Correlation } \\
\text { Techniques }\end{array}$ & FY12 & $\begin{array}{l}\text { Describes the measurements } \\
\text { performed at the NDSTC at } \\
\text { Y-12 National Security } \\
\text { Complex }\end{array}$ & 6 \\
\hline $\begin{array}{l}\text { (U) Progress of the } \\
\text { Measurements in the } \\
\text { Nuclear Detector and } \\
\text { Sensor Test Center at } \\
\text { the Y-12 National } \\
\text { Security Complex }\end{array}$ & FY12 & $\begin{array}{l}\text { Describes the progress of the } \\
\text { measurements mid-FY } 12\end{array}$ & 6 \\
\hline $\begin{array}{l}\text { (U) Statement of Y-12 } \\
\text { Support for } \\
\text { Measurements in } \\
\text { the Nuclear Detector } \\
\text { and Sensor Test } \\
\text { Center }\end{array}$ & FY12 & $\begin{array}{l}\text { Describes the } Y-12 \text { support for } \\
\text { the measurements }\end{array}$ & 6 \\
\hline
\end{tabular}




\section{TeChNique Merit AND Future Work}

In summary, the modeling and measurements indicate that the technique of activeinduced time correlations can be a useful method for determining enrichment of shielded bulk uranium. The highest value suite of measurements enables a comparison of the measurement observables (neutron singles and coincidences) between a $93.2 \mathrm{wt} \% \mathrm{HEU}$ casting and a DU casting. The castings were measured bare or within one or more $1.2 \mathrm{~cm}$ layers of DU shielding with a version of the ORNL NMIS shown in Figure 1. A comparison of the observables for $2.5 \mathrm{~cm}$ of DU shielding $(36 \mathrm{~kg})$ is shown in Figure 2. The simulated induced fissions as a function of position for a time-tagged and directionally tagged cone of neutrons are shown on the left, while the measured time distributions of neutrons and gamma rays correlated to each $14 \mathrm{MeV}$ neutron are shown on the right. The distinguishing signature in the right of the time distributions represents the number of coincident neutrons from fission chains. For this amount of DU shielding, the $93.2 \mathrm{wt} \% \mathrm{HEU}$ casting signature is $75 \%$ stronger than the DU casting signature for a 10-min measurement.

These data were used to determine if the radiation transport simulations could predict the measurement observables for up to three DU shielding configurations. The point kinetics theory of fission chain propagation was extended to incorporate external sources such that the multiplication could be estimated from the observed singles and coincidences (i.e., doubles) with a known efficiency. For these measurements, a ${ }^{252} \mathrm{Cf}$ source was used to estimate the fission neutron efficiency. Because this efficiency becomes increasingly less valid with increasing DU shielding, the fast neutron transmission image was used to predict the decrease in efficiency. Then Monte Carlo N-Particle (MCNP) was used to calculate the multiplication using the KCODE method. The comparison between multiplications inferred from the measurements and MCNP are shown in Table 2. The results agree within uncertainty for a 2 min measurement, indicating that the multiplication comparison worked well for this configuration.

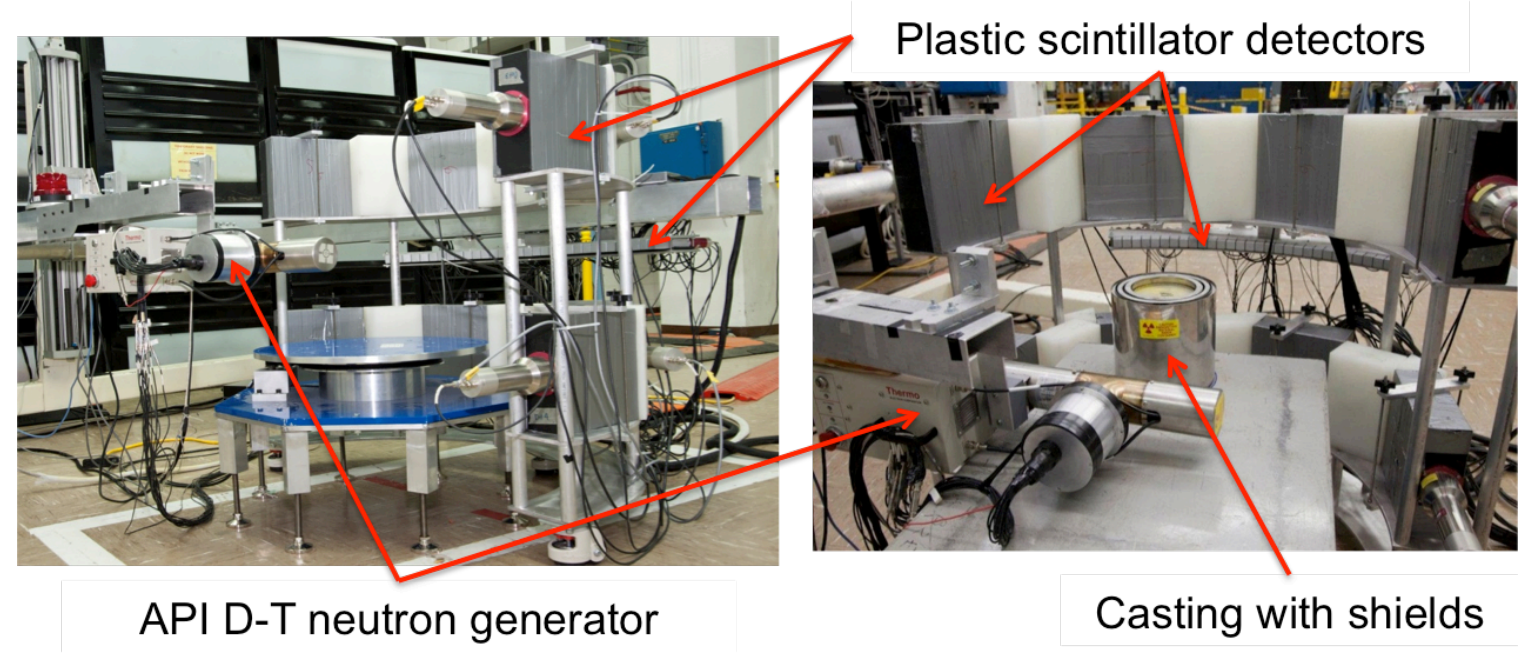

Figure 1. Nuclear Materials Identification System (NMIS) setup at the Nuclear Detector and Sensor Test Center with a DU casting and multiple DU shields. 

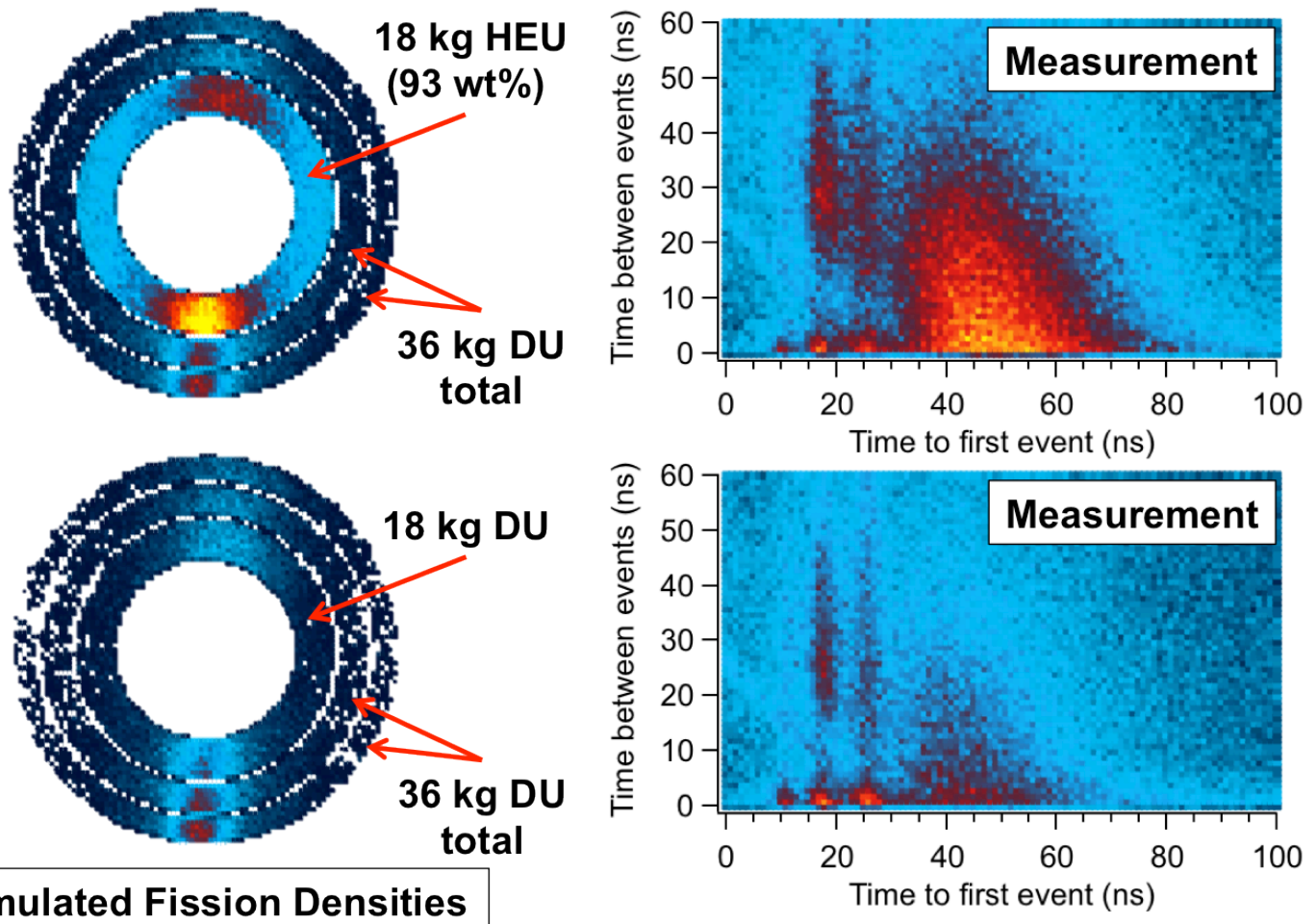

\section{Simulated Fission Densities}

Figure 2. Demonstration of the sensitivity of the active-induced neutron coincidences to enrichment using a $93.2 \mathrm{wt} \%$ HEU casting, DU casting, and $2.5 \mathrm{~cm}$ DU shielding.

Table 2. Comparison of measured multiplications for a $93.2 \mathrm{wt} \% \mathrm{HEU}$ casting, DU casting, and various amounts of DU shielding. Uncertainties represent the $3 \sigma$ error bar.

\begin{tabular}{|lccccc|}
\hline \multicolumn{1}{|c}{ Shield } & Measured $M$ & MCNP $M$ & Measured $M$ & MCNP $M$ & $\begin{array}{c}\text { Corrected } \\
\text { efficiency }\end{array}$ \\
\hline None & $1.14 \pm 0.07$ & 1.12 & $1.73 \pm 0.07$ & 1.79 & 0.047 \\
\hline $\mathbf{1 . 2}$ cm DU & $1.16 \pm 0.06$ & 1.16 & $2.00 \pm 0.07$ & 2.09 & 0.039 \\
\hline $\mathbf{2 . 5}$ cm DU & $1.15 \pm 0.06$ & 1.19 & $2.22 \pm 0.07$ & 2.26 & 0.032 \\
\hline $\mathbf{3 . 8}$ cm DU & $1.12 \pm 0.06$ & 1.21 & $2.46 \pm 0.07$ & 2.36 & 0.026 \\
\hline
\end{tabular}


The agreement between the modeling and measurement results by comparing the multiplication led to the enrichment determination algorithm flowchart shown in Figure 3. Because there is no obvious formula or set of formulas that can be used for an arbitrary geometry to estimate the enrichment, the iterative algorithm is designed such that the fast neutron transmission image provides the geometry. As a result, only the enrichment in the regions indicating uranium needs to be varied. An algorithm based upon a multiplication comparison runs in less than a minute on a standard laptop computer. Although the computation speed is good for the algorithm, the accuracy is limited by the use of point kinetics when estimating the observed multiplication. For the annuli configuration, the cylindrical symmetry enables the point kinetics assumption to be reasonably good, especially with a correction to the efficiency when shielding is present.

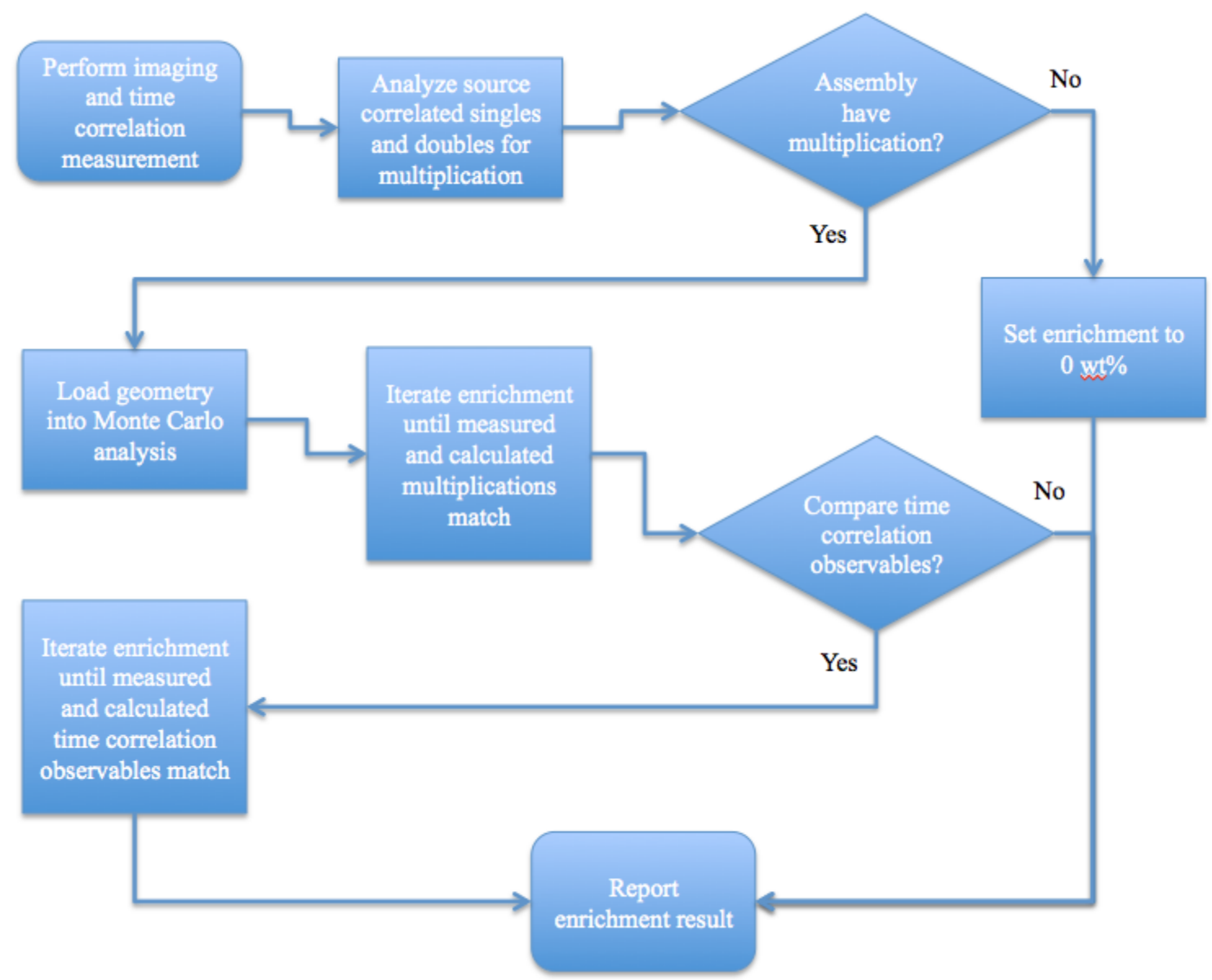

Figure 3. Enrichment determination algorithm flowchart based upon the multiplication.

While the modeling and measurements were particularly focused on EU annuli surrounded by DU annuli, the technique can be applied to arbitrary configurations where the point kinetics assumption is invalid. In applications where only the measured enrichment above some threshold is required, a low enough threshold may be sufficient 
to enable the algorithm in Figure 3 to be used with items with enrichments far from the threshold.

For applications where more accuracy is required, a better approach would be to directly analyze the measured time correlation distributions and model them for comparison. The primary advantage of this approach is the comparison of the directly measured time correlation distributions as opposed to an intermediary observable like the multiplication. The two main challenges with this approach are that (1) the emission energies and multiplicities of the fission neutrons must be modeled with sufficient precision, and (2) the detector response as a function of energy must be well understood. The primary disadvantage of this approach is the increased computational time in comparison to the approach using the multiplication comparison. However, a requirement for excellent accuracy warrants a full three-dimensional calculation. More importantly, ORNL has been unable to accurately predict the measured time correlation distributions with the typically used modeling tool MCNP-PoliMi. An example of the discrepancy is demonstrated for a bare HEU casting in Figure 4. Although some alpha pixel and single detector correlations can be predicted well, others are not predicted well. Thus far, ORNL is still working to understand the nature of the discrepancy. Potential contributors to the discrepancy are insufficient understanding of the detector response and insufficient physics modeling of the neutron energies and multiplicities in MCNP. More recently, the improved fission model FREYA (Fission Reaction Event Yield Algorithm) has been incorporated into MCNP and may allow ORNL to concentrate on improving the detector response model.
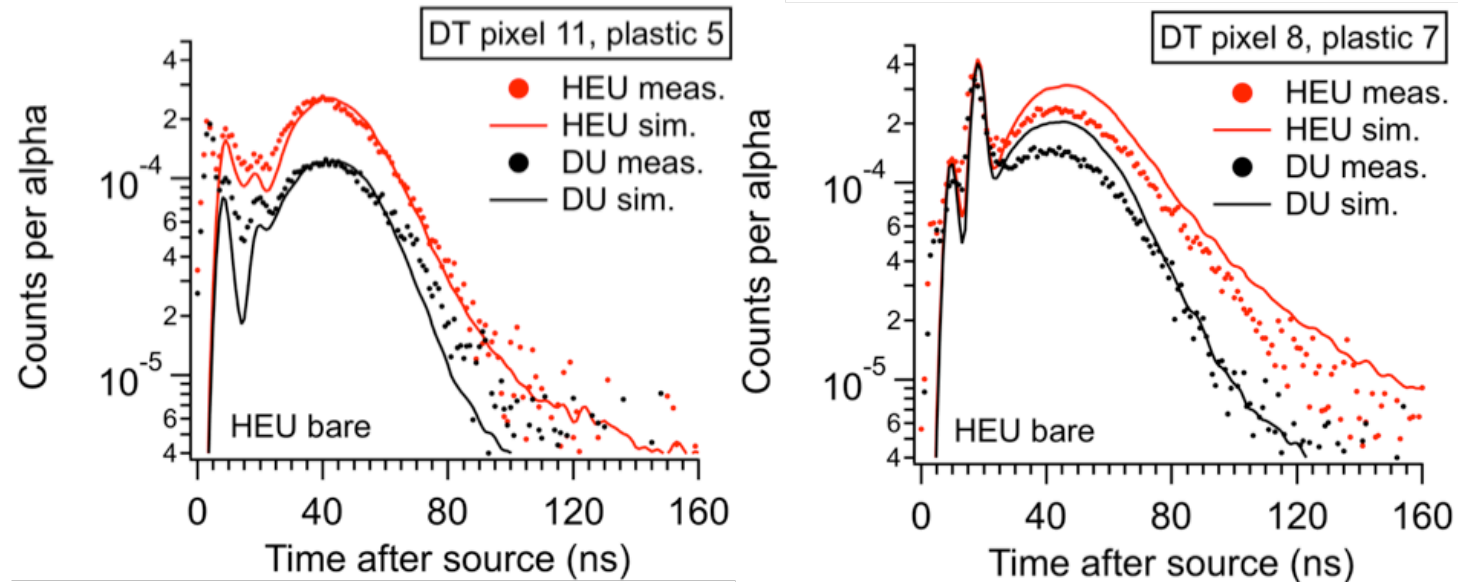

Figure 4. Demonstration of the discrepancy when comparing modeled and measured time correlation distributions.

Overall, active-induced time correlation measurements with an API D-T neutron generator and an array of plastic scintillators enable the determination of enrichment in bulk uranium configurations. As the time correlation observables are degenerate in geometry and enrichment, the active fast neutron image eliminates the degeneracy in geometry. As compared to techniques such as passive gamma spectrometry and delayed neutron precursor counting, the analysis requires an iterative process of executing 
radiation transport code and comparisons to the measurement observables. This process is more complex and typically requires more computational time to generate a result.

The future work may be outlined as:

\section{Perform measurements with enrichments between DU and $93.2 \mathrm{wt} \%$}

In this project, measurements were only performed on a $93.2 \mathrm{wt} \%$ casting and a DU casting. Y-12 attempted to acquire castings of other enrichments, but experienced material acquisition challenges. While the castings that were measured span the space of enrichment, the data represent extrema. The behavior of the time correlation observables has been investigated through predictive modeling only and thus has not been verified. Future users of the NDSTC would benefit with the availability of castings of other enrichments.

\section{Improve the ability to predict the active-induced time correlation distributions}

ORNL identified two areas of effort for improving the ability to predict the activeinduced time correlation distributions: (1) use models that include the most physics for sampling the fission neutron energies and multiplicities, and (2) improve understanding of the detector response model. The first effort can be accomplished with fission modeling codes such as the General Description of Fission Observables or FREYA. FREYA has been incorporated into MCNP6; ORNL would have to transition from MCNP-PoliMi to MCNP6. ORNL expects that the implementation of FREYA into MCNP6 will eliminate the physics modeling as an issue and would then be free to concentrate on improving detector response models. ORNL has experienced challenges in predicting the detector response for the large-area, low-pixel-density plastic scintillators that are typically used with NMIS. ORNL hypothesizes that the light transport is not being modeled sufficiently for the large detectors. The model currently does not account for any spatial dependence for the scattering events within the detector, which produces the light. An important series of experiments would be to obtain a raster

scan of various collimated sources of known energy (e.g., ${ }^{137} \mathrm{Cs},{ }^{252} \mathrm{Cf}$ time-of-flight, etc.) in front of detectors of varying sizes to map the light response. ORNL would also perform simulations with the light transport capability in Geant4, which has been used to develop the high-pixel-density plastic scintillator-based active fast neutron imaging system known as the Advanced Portable Neutron Imaging System.

\section{Develop the algorithms to translate the fast neutron image to a format for use in the radiation transport code}

Using the OpenCV toolkit (computer vision), ORNL successfully developed a code to extract surfaces from the fast neutron image reconstruction and make MCNP surface cards. The next step is the development of a code to make volumes from the surfaces. Although the first version would make MCNP cell (volume) cards, a code that extracts the logical and physical volumes from the image would generally be needed regardless of whether MCNP is used. 


\section{Investigate further improvements to the enrichment determination algorithm}

ORNL has identified two efforts that could potentially lead to improvements to the enrichment determination algorithm. The total number of active-induced fission chain singles and doubles is a function of the multiplication and the probability that a source neutron will induce the first fission in the chain. The multiplication dependence on enrichment has a complex geometrical dependence related to the propagation of fission neutrons through the geometry. In contrast, the probability of first fission dependence on enrichment manifests as the relative difference in fission cross-section for ${ }^{235} \mathrm{U}$ and ${ }^{238} \mathrm{U}$ at $14 \mathrm{MeV}$ (approximately a factor of two). For those tagged neutrons traversing the geometry along a particular known path, the fast neutron image provides the information needed to calculate the probability of first fission. Using the point kinetics formalism, the singles and doubles can be used to estimate the multiplication and therefore the coupling probability. The first effort would investigate the sensitivity of the coupling probability to enrichment and determine if the enrichment can be extracted, for example, using a single expression. However, the paths through an item with a few or more mean free paths can complicate the extraction of the enrichment. As a result, the second effort would be to explore image reconstruction techniques that reconstruct the coupling probability as a function of position in the item. This effort would enable a re-assessment of the computational time required for the enrichment determination algorithm.

\section{Assess the application of the enrichment determination algorithm on classified uranium shapes}

ORNL has sufficient data with classified shapes with which to assess the application of the enrichment determination algorithm. 